Annuaire suisse de politique de développement

Société de l'information et coopération internationale

\title{
Pour une approche africaine des technologies de l'information
}

Sylvestre Ouédraogo

\section{(e) OpenEdition}

1 Journals

Édition électronique

URL : http://journals.openedition.org/aspd/529

DOI : $10.4000 /$ aspd. 529

ISSN : 1663-9669

Éditeur

Institut de hautes études internationales et du développement

Édition imprimée

Date de publication : 1 novembre 2003

Pagination : 31-33

ISSN : 1660-5934

\section{Référence électronique}

Sylvestre Ouédraogo, «Pour une approche africaine des technologies de l'information », Annuaire suisse de politique de développement [En ligne], 22-2 | 2003, mis en ligne le 17 mars 2010, consulté le 08 septembre 2020. URL : http://journals.openedition.org/aspd/529 ; DOI : https://doi.org/10.4000/ aspd.529 


\title{
Pour une approche africaine des technologies de l'information
}

\author{
Sylvestre Ouédraogo*
}

es nouveaux concepts de développement sont légion et suivent souvent des modes. Il n'empêche qu'ils peuvent avoir des conséquences très dommageables sur les politiques et les actions entreprises sous leur couvert. La «fracture numérique» n'y échappe pas.

Comme partout, les outils de communication ont toujours existé en Afrique: tam-tam, marche à pied, courrier ou téléphone... Pourtant, l'avènement des TIC a contraint les moins nantis à tenter des sauts périlleux pour être en phase avec les autres parties du monde. C'est de là que vient l'idée de «fracture numérique».

Les avantages des nouveaux outils sont martelés par les médias et deviennent incontournables dans l'esprit des gens. On oublie alors vite la différence entre le potentiel des nouvelles technologies et les besoins réels des populations. Au contraire, des schémas sont construits pour montrer tout ce que les TIC peuvent apporter à l'Afrique, comme si l'outil pouvait remplacer l'objectif de développement. Pourtant, même en équipant chaque ménage africain d'une ligne à haut débit, les indicateurs de développement n'évolueraient pas. $\mathrm{Au}$ contraire, ceux qui peuvent avoir accès à cette technologie sans aide externe en profiteraient davantage, ce qui accentuerait encore la fracture sociale.

L'identification des besoins est un préalable important pour éviter les dérives. La différence entre l'efficacité dans l'usage des outils et leur efficience est souvent très grande. Par exemple, en utilisant un programme informatique, je serai peut-être efficace et n'aurai plus besoin de mes trois agents qui assuraient cette fonction. Je peux cependant être amené à ne pas utiliser l'outil pour sauver leurs postes si nous avons certaines affinités sociales.

Les inégalités d'accès aux TIC doivent donc être analysées avec soin afin d'investir dans des domaines porteurs. Ce n'est pas l'inégalité d'accès, mais le désir des Africains d'expérimenter ou non ces nouveaux outils qui déterminera le succès des actions.

Les politiques de rattrapage technologique, telles qu'elles sont défendues par les organisations internationales, sont similaires à celles qui ont été menées, après les indépendances, par l'introduction de «paquets technologiques» dans l'agriculture. On pensait alors qu'en utilisant des tracteurs et des engrais chimiques, on s'autosuffirait et on exporterait des surplus. 
L'expérience a montré que l'usage de ces paquets technologiques peut aussi aggraver la pauvreté, détériorer les sols et précipiter de milliers de producteurs dans le cercle de l'endettement.

Ces politiques sont donc à prendre avec précaution. Elles s'apparentent parfois à un «pillage» des ressources intellectuelles du continent (stimulation à la création de contenus sans demander l'avis des bénéficiaires, numérisation de documents qui sont davantage consommés au Nord qu'en Afrique) et à un désir d'étendre l'horizon des marchés saturés de l'Occident (Internet est plus mercantile qu'altruiste). Une des preuves de cette tendance est l'innovation très rapide des technologies qui conduit à des ajustements très fréquents et très coûteux. Les machines sont vite obsolètes, les débits en Europe de plus en plus rapides et les contenus de plus en plus lourds à charger, ce qui met les pays du Sud dans une perspective de course sans fin. Il suffirait pourtant aux concepteurs de sites de faire des versions allégées pour permettre d'accélérer l'accès au web. L'accroissement des débits va de pair avec le développement d'effets superflus. C'est ainsi que se créent les fausses fractures, synonymes de faux problèmes à résoudre.

La réduction arithmétique de la fracture numérique est donc une utopie. Si elle était possible, elle serait tout simplement un gaspillage de ressources: un téléphone par ménage en Occident peut être une priorité; en Afrique subsaharienne, un téléphone par village serait un vrai succès. En cherchant des comparaisons sans travailler sur des critères propres à chaque région, on ne fait qu'aggraver la fracture, au profit de certains. Les marchands de logiciels et de matériels ont de beaux jours devant eux, puisqu'ils n'encouragent pas la création, la production et la maintenance au niveau local.

La tendance à valoriser l'usage des TIC contribue à inhiber les outils traditionnels et les savoirs locaux. Par exemple, ce n'est pas par Internet qu'un paysan ira apprendre des techniques agricoles. Ce qui l'intéresse, c'est d'écouler ses produits. Ce sont les effets d'incitation (prix des produits intéressants, facilité d'écoulement des produits...) qui influent sur l'usage et la recherche de technologies adaptées, et non l'inverse. Si l'outil ne permet pas d'augmenter le chiffre d'affaires, il sera abandonné. On n'a jamais fait de publicité pour que les analphabètes utilisent la machine à calculer. Pourtant, beaucoup de petits commerçants trouvent leur compte dans les calculettes et les téléphones portables.

Il est donc important de nuancer les potentiels des TIC en insistant sur des secteurs qui ont déjà un certain niveau de technicité, pour ensuite accompagner prudemment les autres secteurs dans ce processus. En Occident, les TIC se sont diffusées en partant de secteurs stratégiques comme la défense et la recherche. Ce n'est qu'ensuite qu'elles ont été adoptées par les entreprises et enfin par les ménages. En Afrique, l'urgence des besoins est telle que l'on a tendance à tout renverser: on essaie de voir les applications possibles partout tout en oubliant de demander l'avis des usagers potentiels et en feignant d'ignorer qu'ils ont des technologies alternatives. C'est comme si on ignorait que l'Afrique communiquait avant les TIC. 
Les ressources de la coopération internationale diminuent. Le vent de la libéralisation aidant, le secteur privé est appelé à jouer un rôle important dans le développement des TIC. Pourtant, l'exemple du Burkina Faso montre que les discours ne prennent pas en compte le comportement des entrepreneurs. Les entreprises étrangères ne se bousculent pas dans des économies aux risques élevés et au faible pouvoir d'achat. Elles n'ont jamais accepté d'investir dans des infrastructures en Afrique, parce que le taux de rentabilité est inférieur au taux de l'intérêt bancaire. Il est plus rentable de placer son argent dans les circuits financiers (épargne, crédits et spéculation) ou de faire de l'importexport.

En revanche, quand l'Etat met en place des infrastructures, le privé les exploite avantageusement. Par exemple, une fois les possibilités d'ouverture de télécentres publics accordées et les infrastructures assurées par l'office des télécommunications, les centres privés ont fleuri à Ouagadougou. Même en province, lorsque les infrastructures le permettent, les cabines téléphoniques se multiplient. Le constat concernant l'accès à Internet est similaire. C'est le faible débit qui décourage les entreprises à investir dans les TIC.
La coopération internationale pourrait intervenir efficacement pour encourager la construction d'infrastructures nationales, la formation de formateurs et appuyer les structures associatives dans la création de contenus multimédias et de centres sociaux d'accès aux TIC.

Les infrastructures de base peuvent être financées par les budgets nationaux, ce qui ferait naître un sentiment d'appartenance nationale. Il suffirait tout simplement que la coopération internationale aide les pays à effacer leurs dettes qui les contraignent à couper dans les investissements importants et à compter sur l'aide internationale. Au niveau de la formation des formateurs, la coopération internationale peut aider à la création de centres de formation régionaux afin que l'appropriation soit effective; sinon, elle ne sera que de façade et une dépendance continuera d'exister. Le secteur des ONG peut aider à créer des contenus et à influer sur les usages et les pratiques, grâce à la création et à l'animation de réseaux impliquant des acteurs multiples.

Tout cela permettrait de créer un esprit «nouvelles technologies» proprement africain et de développer les TIC en fonction des intérêts et des conditions spécifiques du continent. 